\title{
Study of the sign problem in canonical approach
}

\section{Ryutaro Fukuda}

Department of Physics, The University of Tokyo

Bunkyo-ku, Tokyo 113-0033, Japan

RIKEN BNL Research Center, Brookhaven National Laboratory, Upton, NY 11973, USA

E-mail: jr-fukuda@nt.phys.s.u-tokyo.ac.jp

\section{Atsushi Nakamura}

RCNP, Osaka University, Osaka567-0047, Japan

Nishina Center, RIKEN, Wako, Saitama 351-0198, Japan

School of Biomedicine, Far Eastern Federal University, Vladivostok, 690950 Russia

E-mail: atsushi@rcnp.osaka-u.ac.jp

\section{Shotaro Oka}

Institute of Theoretical Physics, Department of Physics, Rikkyo University

Toshima-ku, Tokyo 171-8501, Japan

E-mail: okasho-hato@rikkyo.ac.jp

\section{Asobu Suzuki}

Graduate School of Pure and Applied Sciences, University of Tsukuba

Tsukuba, Ibaraki 305-8571, Japan

E-mail: suzuki@het.ph.tsukuba.ac.jp

\section{Yusuke Taniguchi}

Graduate School of Pure and Applied Sciences, University of Tsukuba

Tsukuba, Ibaraki 305-8571, Japan

E-mail: tanigchi@het.ph.tsukuba.ac.jp

It is known that the sign problem emerges as a complex phase of the canonical partition function in canonical approach. We confirmed that the origin of the complex phase is breaking of the charge conjugation with a simple model. The main purpose of this study is suppressing the complex phase even at low temperature. We realized it by increasing the number of gauge field configurations. In particular, the complex phase was suppressed less than $\pi / 2$ in $1 \sigma$ for $N_{B}<7$ at $T=0.81 T_{c}$

34th annual International Symposium on Lattice Field Theory

24-30 July 2016

University of Southampton, UK

\footnotetext{
*Speaker.
} 


\section{Introduction}

One of the main motivation of the finite density QCD is exploring the QCD phase diagram, and confinement/deconfinement phase transition is a hot topic for both theory and experiment. The biggest failure for the numerical simulation is the sign problem which is a series of problem accompanying the complex action at non-zero chemical potential. There are a lot of candidates which beat the sign problem, complex Langevin method, Lefschetz thimble method, multi-parameter reweighting method and canonical approach. We chose the canonical approach in which we can perform the Monte Carlo simulation.

It is well known that the sign problem emerges as a complex phase of the canonical partition function. The canonical partition function should take a real value, however, it has a non-zero complex phase for each configurations. The main motivation of this paper is studying about some properties of the complex phase, the origin of the complex phase, temperature dependence and baryon number dependence.

The canonical partition function is given by Fourier transformation of the grand canonical partition function as in eq.(1.1), and is equivalent to the fugacity expansion which is givin by eq.(1.2). The canonical partition function is a basic quantity in the canonical approach[1].

$$
\begin{aligned}
& Z_{\text {can. }}(T ; V, N)=\frac{1}{2 \pi} \int_{-\pi}^{\pi} d \frac{\mu}{T} Z_{G . C .}(T, i \mu ; V) e^{-i \frac{\mu}{T} N} \\
& Z_{G . C .}(T, \mu ; V)=\sum_{N} e^{\frac{\mu}{T} N} Z_{\text {can. }}(T ; V, N)
\end{aligned}
$$

When we use the path integral representation of the grand canonical partition function and the reweighting technique, we get an expression of the canonical partition function.

$$
\begin{aligned}
Z_{c a n .}(T ; V, N) & =\frac{1}{2 \pi} \int_{-\pi}^{\pi} d \frac{\mu}{T} \int \mathscr{D} U \frac{\operatorname{det} D(i \mu)}{\operatorname{det} D(0)} e^{-i \frac{\mu}{T} N} \operatorname{det} D(0) e^{-S_{g}} \\
& =\left\langle\frac{1}{2 \pi} \int_{-\pi}^{\pi} d \frac{\mu}{T} \frac{\operatorname{det} D(i \mu)}{\operatorname{det} D(0)} e^{-i \frac{\mu}{T} N}\right\rangle_{\mu=0}
\end{aligned}
$$

In the second line, we introduced a new symbol $\langle\cdots\rangle_{\mu=0}$ which means a gauge field average with respect to zero chemical potential. We have to calculate $\operatorname{det} D(i \mu) / \operatorname{det} D(0)$ for many times to perform the Fourier transformation, and such calculation is heavy from the numerical point of view. The winding number expansion method[2] gives us an easy expression of $\operatorname{det} D(i \mu) / \operatorname{det} D(0)$.

$$
\frac{\operatorname{det} D(\mu)}{\operatorname{det} D(0)}=e^{\operatorname{Tr}\{\log (1-\kappa Q)\}}=\exp \left(-\sum_{n=1} \frac{\kappa^{n}}{n} \operatorname{Tr}\left\{Q^{n}\right\}\right)=\exp \left(\sum_{k} W_{k} e^{\frac{\mu}{T} k}\right)
$$

Coefficients $\left\{W_{k}\right\}_{k}$ are defined throughout hopping terms $Q$ defined in the eq.(1.6). Moreover, we can show that the coefficients $\left\{W_{k}\right\}_{k}$ do not depend on the chemical potential. Thus, once we get them we can evaluate $\operatorname{det} D(i \mu) / \operatorname{det} D(0)$ without a direct computation of the determinant.

$$
\begin{aligned}
Q & =\sum_{i=1}^{3}\left(Q_{i}^{(+)}+Q_{i}^{(-)}\right)+e^{\mu} Q_{4}^{(+)}+e^{-\mu} Q_{4}^{(-)} \\
Q_{v}^{(+)}(x, y) & :=\left(1-\gamma_{v}\right) U_{v}(x) \delta_{x, y-\hat{v}} \\
Q_{v}^{(-)}(x, y) & :=\left(1+\gamma_{v}\right) U_{v}^{\dagger}(x-\hat{v}) \delta_{x, y+\hat{v}}, v=1 \cdots 4
\end{aligned}
$$




\section{Lattice setup}

We used the Iwasaki gauge action and 2-flavors Wilson Clover action. Our calculation was done at two simulation points to discuss about a temperature dependence of the canonical partition function. Parameters are listed in the table below. We took the quark mass in heavy region $m_{\pi} / m_{\rho} \sim 0.7$, because the winding number expansion method (1.5) is equivalent to the hopping parameter expansion. We performed the hopping parameter expansion to 480th order. Multiprecision calculation is needed to perform the Fourier transformation. This fact is suggested in [2][3].

\begin{tabular}{|l|c|r|r|r|r|}
\hline$\beta$ & $\kappa$ & $C s w$ & $T / T_{c}$ & $m_{\pi} / m_{\rho}$ & Lattice \\
\hline 1.5 & 0.131 & 1.1 & 0.81 & $0.756(13)$ & $8^{3} \times 4$ \\
1.9 & 0.125 & 1.1 & 1.68 & $0.714(15)$ & $8^{3} \times 4$ \\
\hline
\end{tabular}

Table 1: Parameters used in this study. We took two temperature, below $T_{c}$ and above $T_{c}$, to discuss how the complex phase depends on the temperature.

\section{Origin of the complex phase}

Let us discuss about the expression of the canonical partition function more carefully to find what the origin of the complex phase is. The canonical partition function is given by following expression.

$$
Z_{\text {can. }}(T ; V, N)=\frac{3}{2 \pi} \int_{-\pi / 3}^{\pi / 3} d \frac{\mu}{T}\left\langle\frac{\operatorname{det} D(i \mu)}{\operatorname{det} D(0)}\right\rangle_{\mu=0} e^{-i \frac{\mu}{T} N},
$$

where we used the $Z_{3}$ center symmetry to rewrite the integration range of the Fourier transformation. We pay attention to two properties of this expression. The first one is that the determinant of the Dirac operator $\langle\operatorname{det} D(i \mu) / \operatorname{det} D(0)\rangle_{\mu=0}$ takes a real value because the chemical potential is pure imaginary. The second one is the integration range is symmetric. We can expect from these facts that breaking of charge conjugation $\langle\operatorname{det} D(i \mu) / \operatorname{det} D(0)\rangle_{\mu=0} \neq\langle\operatorname{det} D(-i \mu) / \operatorname{det} D(0)\rangle_{\mu=0}$ makes the complex phase. To justify our claim, we introduced a model in which the grand canonical partition is given as follows.

$$
Z_{G . C .}(T, i \mu ; V)=e^{-a\left(\frac{\mu}{T}\right)^{2}+b\left(\frac{\mu}{T}\right)}
$$

This model has two parameters, $a$ means a gaussian slope and $b$ means an asymmetricity with respect to the chemical potential. Numerical results of the grand canonical partition function can be reproduced in our integration range $-\pi / 3<\mu / T<\pi / 3$ as in figure 1 , the red points shows the numerical results and the green solid line shows our model. Moreover, the canonical partition function can be written down as follows in our model.

$$
Z_{\text {can. }}(T ; V, N)=(\text { const. }) e^{\frac{(b-i N)^{2}}{4 a}} \int_{-\pi / 3}^{\pi / 3} d x e^{-a\left(x-\frac{b-i N}{2 a}\right)^{2}}
$$




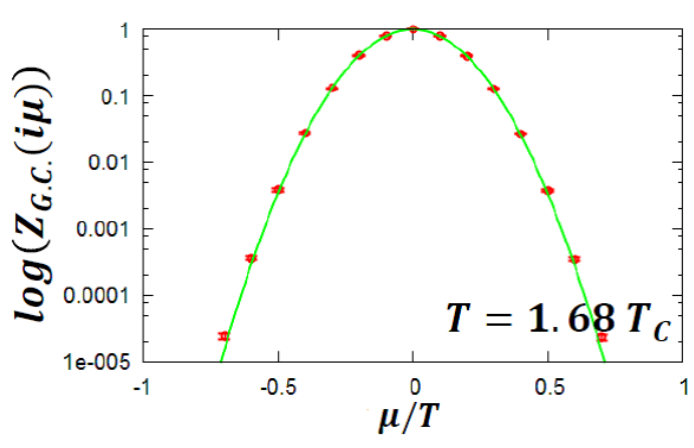

Figure 1: We can fit the numerical data by the simple model given in eq.(3.2). The numerical data is shown as red points and the fit is shown as green line.

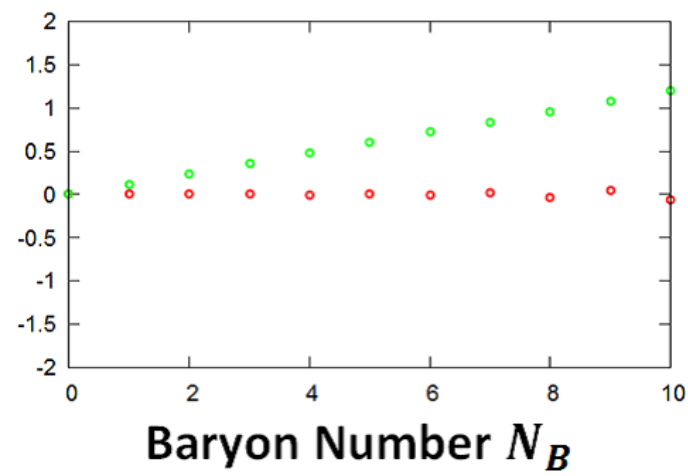

Figure 2: Evaluation of the complex phase. Red points shows the third factor of eq.(3.3) and green points shows the second factor of eq.(3.3).

We can say that the third factor of this expression is almost Gaussian integration and the second factor mainly contributes to the complex phase. In reality, the contribution of the second factor is larger than the third factor as shown in the figure 2. Therefore, the complex phase of the canonical partition function can be approximated by

$$
\arg \left(Z_{\text {can. }}(T ; V, N)\right) \sim-\frac{b}{2 a} N
$$

in this model. According to the winding number expansion method[2] the complex phase can be evaluated

$$
\arg \left(Z_{\text {can. }}(T ; V, N)\right) \sim-\arg \left(W_{1}\right) N
$$

where the coefficient $W_{1}$ is same as previous one. Both results tell us that the complex phase is proportional to $-N$. We confirmed this property for one configuration in the figure 3 and 4 . The figure 3 shows the difference between $\log Z_{G . C .}(i \mu)$ and $\log Z_{G . C .}(-i \mu)$, thus, the asymmetricity. The figure 4 shows the complex phase of the canonical partition function. We looked how the breaking of the charge conjugation contributes to the complex phase in this section. We will discuss about the temperature dependence of the complex phase by using this simple model.

\section{Temperature dependence of the complex phase}

It is known that the sign problem in other words behavior of the complex phase is getting worse at low temperature. We checked the same property in the figure 5 . The phase is consistent with 0 and has small error bars, thus, the sign problem is under control at $T=1.68 T_{c}$. However, the phase is beyond $\pi / 2$ even for small baryon number at $T=0.81 T_{c}$. The figure 6 and 7 show temperature dependence of the asymmetricity. We can see that the asymmetricity takes almost the same order, but the fluctuation is slightly large at low temperature and the Gaussian slope has a large dependence as shown in the figure 8 and 9. The Gaussian slope is smaller at low temperature. We estimated the Gaussian slope, $a \sim 0.807$ at $T=0.81 T_{c}$ and $a \sim 22.7$ at $T=1.68 T_{c}$ by fitting 


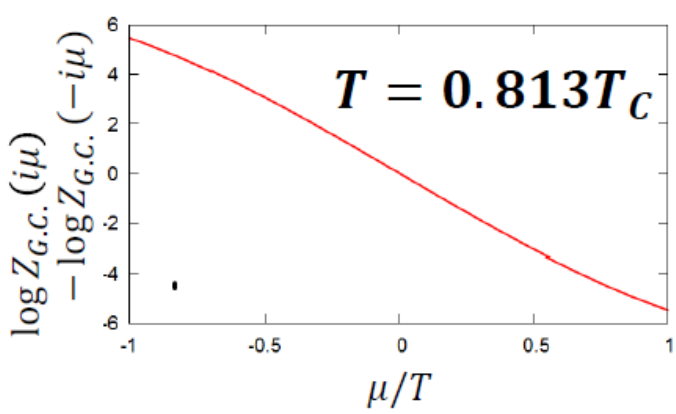

Figure 3: Evaluation of the asymmetricity. The parameter $b$ takes negative value in terms of our simple model.

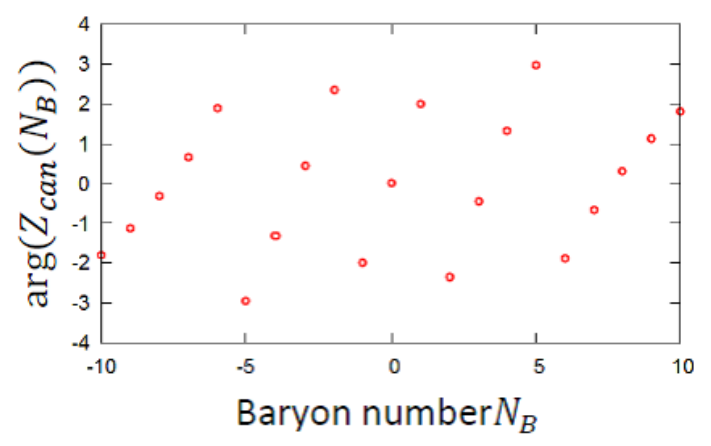

Figure 4: The complex phase has a positive slope. This fact is consistent with our evaluation of the complex phase (3.4).

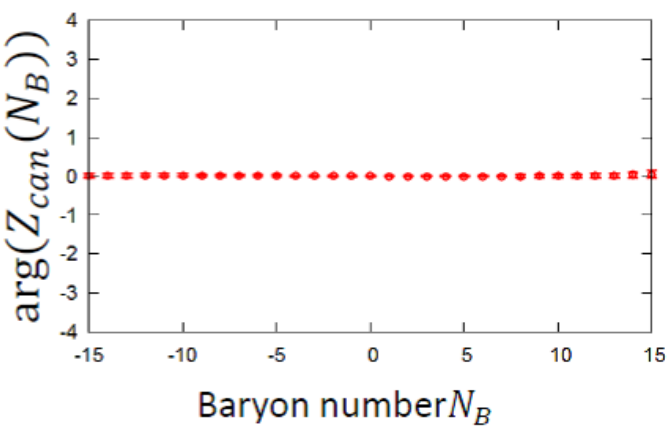

Figure 5: Temperature dependence of the complex phase. Left figure shows the numerical results at $T=$ $0.81 T_{c}$ and right figure shows at $T=1.68 T_{c}$.
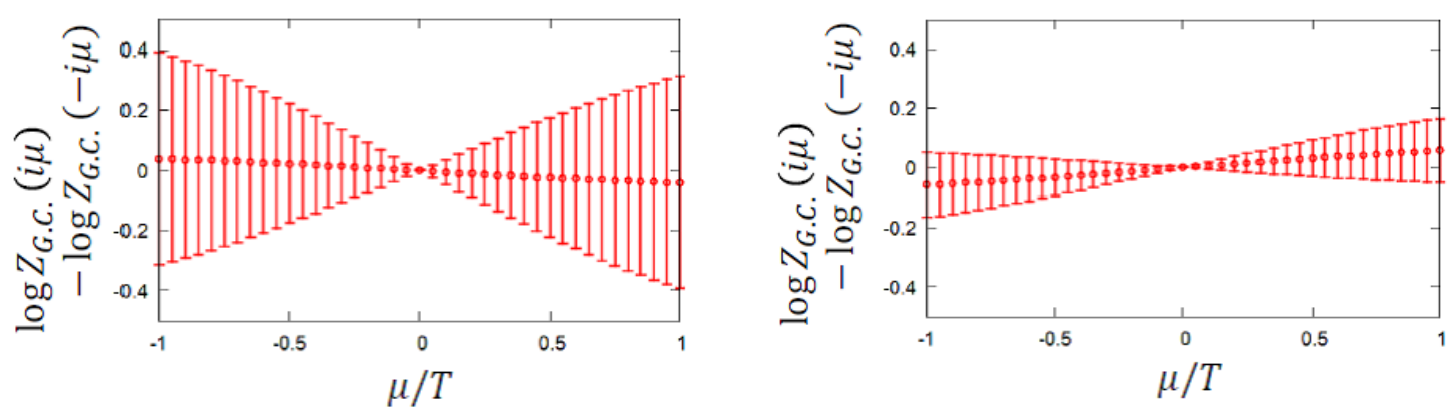

Figure 6: Breaking of charge conjugation at $T=$ Figure 7: Breaking of charge conjugation at $T=$ $0.81 T_{c}$. Fluctuation is slightly larger than $T=1.68 T_{c}$. $1.68 T_{c}$. 
the numerical data. Since the phase can be approximated $-b N / 2 a$, we can say that the fluctuation of the complex phase is amplified at low temperature and is suppressed at high temperature. We

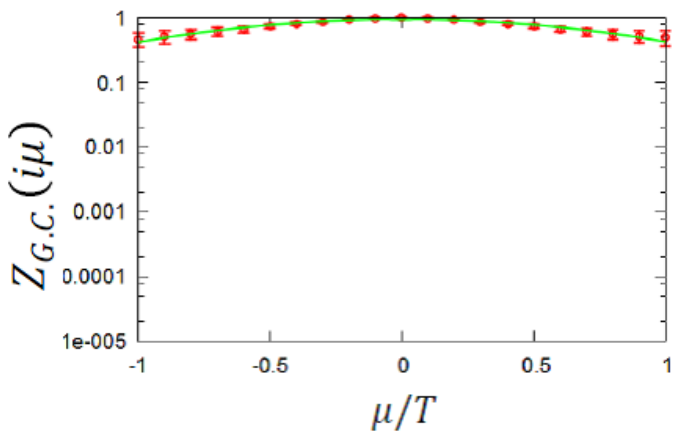

Figure 8: Estimation of the Gaussian slope at $T=$ Figure 9: Estimation of the Gaussian slope at $T=$ $0.81 T_{c} . a \sim 0.807$ in this parameter.

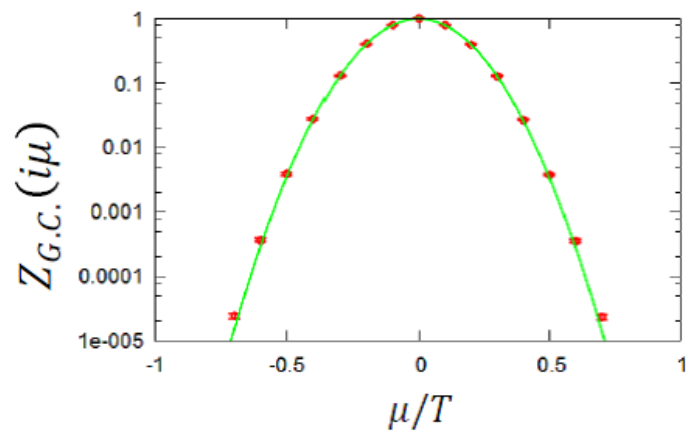

$1.68 T_{c} . a \sim 22.7$ in this parameter.

can see that the numerical results of the complex phase are also proportional to the baryon number, and such dependence can be explained by our model. Our model gave us some clues to understand the properties of the complex phase, temperature dependence and baryon number dependence. In the next section, we will try to suppress the complex phase even at low temperature from these knowledge.

\section{To suppress the complex phase}

We checked in the above sections that the breaking of the charge conjugation makes the complex phase. Of course, such contribution does not emerge analytically. The grand canonical partition function is symmetric and the complex phase is 0 . Since we cannot change the Gaussian slope $a$, we must fight the complex phase at the asymmetricity $b$. Thus, the most naive expectation is that the complex phase is suppressed by increasing the statistics. We checked that the distribution of the gauge field become symmetric when we increase the number of the gauge field configurations in the figure 10. Our model tells us that the complex phase will be suppressed with the decrease of
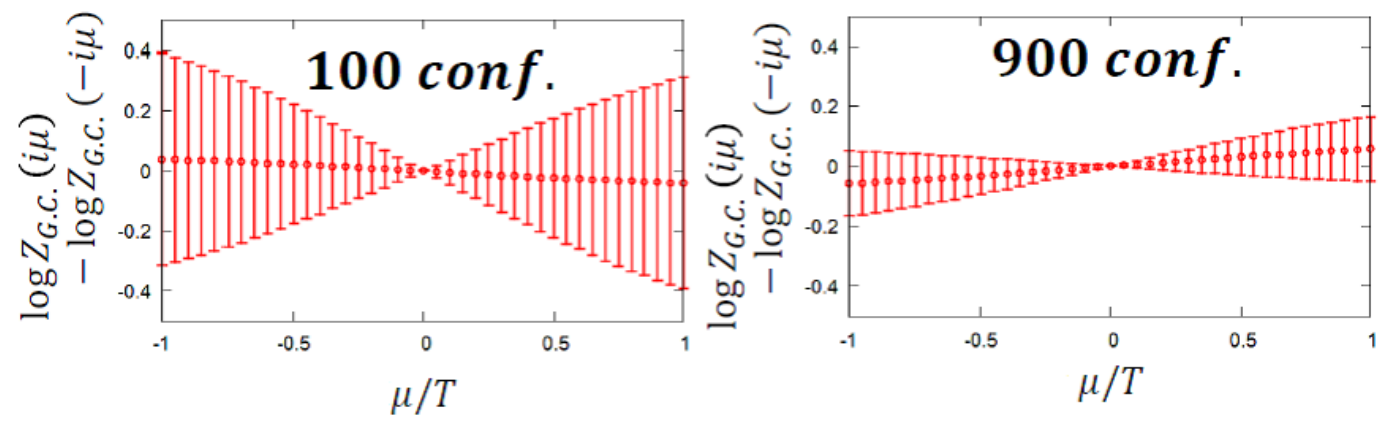

Figure 10: Configuration number dependence of the asymmetricity at $T=0.81 T_{c}$. We made the statistics 9 times and the fluctuation was suppressed. 
the asymmetricity $b$. Thus, we can expect that the complex phase itself will be improved by taking 900 configurations. The numerical results are shown in the figure 11 . The complex phase is surely suppressed with increasing the configurations. In particular, when we take 900 configurations the complex phase is less than $\pi / 2$ in $1 \sigma$ for small baryon number $N_{B}<7$.
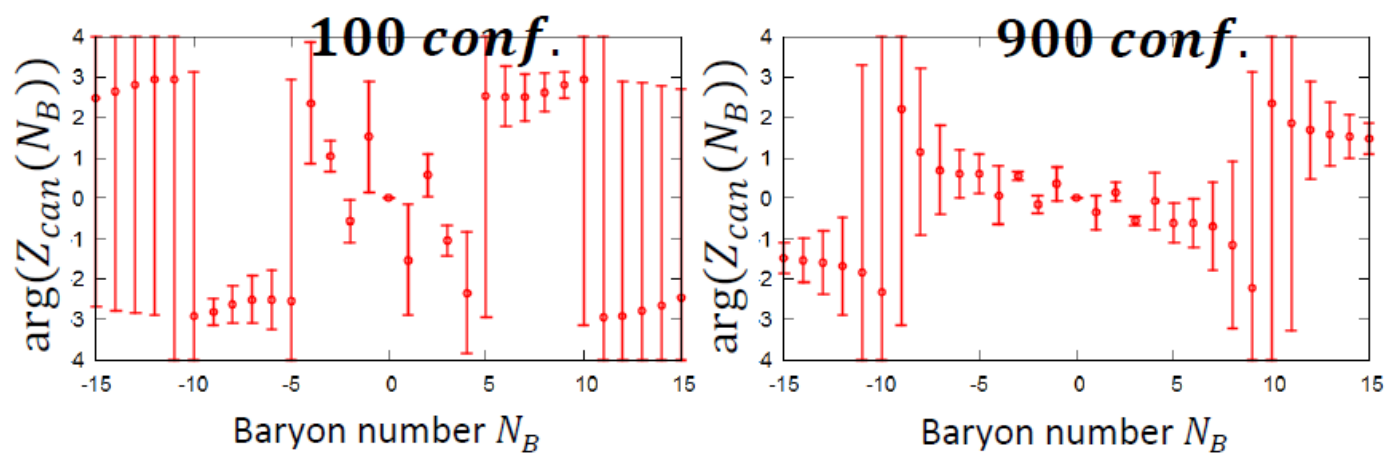

Figure 11: Configuration number dependence of the complex phase at $T=0.81 T_{c}$. It is suppressed less than $\pi / 2$ in $1 \sigma$ for $N_{B}<7$

\section{Conclusion}

The sign problem emerges as the complex phase of the canonical partition function in the canonical approach. At first, we discussed about the origin of the complex phase. The expression of the canonical partition function tells us that the breaking of the charge conjugation makes the complex phase. We introduced a simple model to justify our claim and it worked well to explain some properties of the complex phase. We tried to suppress the complex phase for small baryon number by increasing the number of configurations at the end of this study. However, we must study the volume dependence of it to discuss the sign problem. It is a future work of our study.

\section{Acknowledgement}

This work is done for Zn Collaboration. We would like to thank V. Bornyakov, D. Boyda, V. Goy, A. Molochkov, A. Nikolaev and V. Zakharov. This work is supported by the Large Scale Simulation Program No.14/15-19 (FY2014) of High Energy Accelerator Research Organization (KEK). This work is supported in part by Grants-in-Aid of the Ministry of Education (Nos. 26610072. 24340054, 22540265). This work is in part based on Bridge++ code (http://suchix.kek.jp/bridge/Lattice-code/).

\section{References}

[1] A. Hasenfratz and D. Toussaint, Nucl. Phys. B 371, 539 (1992).

[2] A. Li, A. Alexandru, K. F. Liu and X. Meng, Phys. Rev. D 82, 054502 (2010).

[3] R. Fukuda, A. Nakamura, and S. Oka, Phys. Rev. D 93, 094508 (2016).

[4] A. Nakamura, S. Oka and Y. Taniguchi, Pos (Lattice2014) 198.

[5] A. Nakamura, S. Oka and Y. Taniguchi, JHEP02 (2016) 054. 